\title{
Nierenbiopsie bei Lupus noch zeitgemäß?
}

Auf diese Frage gab es beim DGRh-Kongress ein klares „Ja“. Beim Lupus ist die Indikation bei Verdacht auf eine Nierenbeteiligung gegeben, wenn Nierenfunktion und Urindiagnostik auffällig sind und sich diagnostische, prognostische oder therapeutische Implikationen ergeben.

Allerdings gibt es einige wenige Gründe für den Verzicht auf eine Biopsie. Dies sind laut Prof. Dr. Stefan Weiner, II. Medizinische Abteilung, Krankenhaus der Barmherzigen Brüder, Trier, Schrumpfnieren, eine Situation mit zahlreichen Nierenzysten im Punktionsgebiet oder die folgenden Kontraindikationen:

- unkontrollierter Bluthochdruck,

- Gerinnungsstörung, ausgeprägte Thrombozytopenie, nicht absetzbare Thrombozytenaggregationshemmer oder Antikoagulanzien,

- unkooperativer Patient.

Bei Patienten unter ASS muss die Behandlung vor einer Nierenbiopsie mindestens 7 bis 8 Tage ausgesetzt werden.

\section{Wertvolle diagnostische, prognostische und therapeutische Hinweise}

Warum die Nierenbiopsie beim systemischen Lupus erythematodes (SLE) weiterhin einen wichtigen Stellenwert hat, erläuterte Weiner in der Sitzung „Organmanifestationen rheumatischer Erkrankungen - ist die Biopsie noch zeitgemäß". Zum einen fordern die aktuellen Klassifikationskriterien von EULAR (European League Against Rheumatism) und ACR (American College of Rheumatology) für den SLE, dass bei Nachweis einer Proteinurie der Nierenbeteiligung histologisch nachgegangen werden soll.
Wird histologisch eine proliferative oder diffuse Lupusnephritis nachgewiesen, ist dies ein Hinweis auf einen schweren Verlauf und ist wegeweisend für die medikamentöse Therapie. Zum anderen lässt sich laut Weiner anhand der Klinik nicht abschätzen, was bei der Biopsie herauskommt. Daher ist die Nierenhistologie zwingend geboten. Denn die KDIGO-(Kidney Disease: Improving Global Outcomes)-Therapieempfehlungen richten sich klar nach der vorliegenden LupusnephritisKlasse. Beim SLE gibt die Biopsie also wichtige diagnostische, prognostische oder therapeutische Hinweise.

》) Die Nierenbiopsie ermöglicht eine Prognoseabschätzung, welche die Wahl der Therapie beeinflusst

Zudem könne der Nephropathologe in der Biopsie zelluläre von fibrösen Halbmonden unterscheiden und so eine exakte Klassifikation der Lupusnephritis vornehmen, so Weiner. Erstere sprächen für eine proliferative Nephritis, letztere zeigten, dass die Glomeruli nicht mehr zu retten seien. Außerdem könne anhand der Vernarbungen eine tubulointerstitielle Nephritis diagnostiziert werden, die aufgrund der tubulären Atrophie mit einer schlechten Prognose einhergehe.

Schließlich erlaube die Biopsie eine Aussage zu vaskulären Erkrankungen, die ebenfalls prognostisch ungünstig seien. Relativ neu ist laut Weiner, dass eine Lupusnephritis Typ II mit nephrotischem Syndrom mit einer Podozytopathie assoziiert ist. Diese sollte mit einem Calcineurin-Inhibitor (Tacrolimus) behandelt werden, da dieser gut antiproteinurisch wirke.

Weinerts Fazit: Die Nierenbiopsie ist bei Verdacht auf eine Lupusnephritis von diagnostischem Nutzen und erlaubt eine exakte Differentialdiagnose. Zudem ermöglicht sie eine Prognoseabschätzung, welche die Wahl der Therapie beeinflusst. Eine wiederholte Nierenbiopsie kann in folgenden drei Situationen indiziert sein:

- Bei persisitierender Proteinurie, aber gebesserter glomerulärer Filtrationsrate (GFR), um zu entscheiden, ob die Therapie reduziert werden kann.

- Um zu klären, ob es zur Umwandlung in eine andere histologische Klasse gekommen ist

- Bei progredientem GFR-Verlust zur Beantwortung der Frage, ob eine aggressive Therapie weiterhin sinnvoll ist.

rheuma plus 2020 • 19:12 https://doi.org/10.1007/s12688-01900308-3

C) Springer-Verlag GmbH Austria, ein Teil von Springer Nature 2019 